\title{
Insight into plasticity mechanisms in metallic glasses by means of a Brazilian test and numerical simulation
}

\author{
J.S. Brest ${ }^{\mathrm{a}, \mathrm{b}}$, V. Keryvin ${ }^{\mathrm{a}, *}$, P. Longère ${ }^{\mathrm{b}}$, Y.Yokoyama ${ }^{\mathrm{c}}$ \\ ${ }^{a}$ Université Européenne de Bretagne, Université de Bretagne-Sud, LIMATB EA 4250, Campus de Saint Maudé, BP \\ 92116, 56321 Lorient Cedex, France \\ ${ }^{b}$ Université de Toulouse, ISAE, ICA EA 814, 10 av. E. Belin, BP 54032, 31055 Toulouse Cedex 4, France \\ ${ }^{c}$ Laboratory for Advanced Materials, Institute for Material Research, Tohoku University, Katahira 2-1-1 Aoba-ku \\ Sendai City, Miyagi Prefecture 980-8577, Japan
}

\begin{abstract}
Multiaxial and heterogeneous mechanical experiments, by means of the diametral compression test (Brazilian test), were carried out on Zr-based Bulk Metallic Glass. Attention was notably focused on obtaining the displacement field in the area of interest (large strains) employing a Digital Image Correlation device, in addition to the measurement of the usual global load-displacement curve. In order to reproduce the behaviour of the BMG at stake, several constitutive equations were considered with growing complexity: von Mises, Drucker-Prager (pressure dependence), free volume based model as well as a viscoplastic Coulomb-Mohr type model. The two latter were implemented as user-material in a Finite Element computation code. The results of these investigations including experiments and computational simulations are discussed.
\end{abstract}

Keywords: Metallic glasses; Diametral compression; Digital Image Correlation; Finite Element Modelling; Plasticity; Shear bands; Constitutive models

\section{Introduction}

Bulk metallic glasses (BMG) are a quite recent class of materials that exhibit exceptional mechanical properties including strength, resilience, hardness or fracture toughness as well as large yield strains $[1,2,3]$. The mechanisms of deformation, damage and failure of these materials must be fully identified for designing compositions of metallic glasses even more resistant and ductile. The numerical prediction of the mechanical resistance for these new materials requires an advanced modelling of their behaviour. A better understanding of the plastic deformation (in terms of localised thin shear bands), the consideration of temperature, viscosity and pressure effects are necessary parameters.

Obtained via a rapid solidification from the liquid state, BMG belong to the class of amorphous alloys characterized by the absence of structural long range ordering. Their macroscopic mechanisms of plasticity mainly proceed from the propagation and interaction of very thin shear bands. From the microscopic viewpoint, BMG plasticity does not result

*Corresponding author: vincent.keryvin@univ-ubs.fr

Preprint submitted to Journal of Alloys and Compounds 586 (2014) S236-S241

April 10, 2014 
from the accumulation and motion of dislocations, as it is the case for crystalline alloys, but may be depicted, depending on the authors, by the theories of shear transformation zones (STZ) [4] or free volume (FV) [5]. Starting from the aforementioned models (STZ and FV), several authors have tried to describe the macroscopic response of BMG to various loading cases, see e.g. Schuh et al. [1] and Gao [6] (FV model), respectively. Following another way, Anand and Su (AS model) [7] developed a Coulomb-Mohr type constitutive theory to reproduce the elastic-viscoplastic behaviour of BMG.

The present work aims at investigating in a first step the mechanical behaviour on $\mathrm{Zr}$ based BMG by means of a multiaxial and heterogeneous test. BMG exhibiting an apparent brittle behaviour in tension as well as in compression at room temperature, the information, namely plasticity, provided by the usual tension or compression tests on these materials are de facto far from being suitable. To get some insight on plasticity mechanisms, the indentation test is commonly carried out $[8,9,10,11]$, so are bending [12] or constrained tests [13]. There exist other tests, though being less known for this type of material, which may also be of particular interest. Among them is diametral compression tests [14], also called Brazilian test, which is extensively used on brittle materials like concrete,. It allows one to generate a tensile loading in the sample section transversally to the compression axis; it also generates heterogeneous mechanical fields in the sample, some parts of the sample remaining almost free of loading. The present approach differs from previous indentation studies since it allows to record extensive plastic fields during the test in addition to the mean response (the load-displacement curve) and it creates multiaxial stress states different from those underneath an indenter. For the indentation test, they are highly compressive and localised underneath the indenter tip, while for the Brazilian test, they are compressive close to the loading conditions, tensile close to the free boundaries and close to pure shear at the centre.

In a second step, computational simulations employing the engineering Finite Element computation code Abaqus are conducted, involving configurations with different BMG constitutive models. The models which do not belong to the standard model library of the computation code were implemented as user materials. The numerical results are commented and compared to experimental ones.

The material and experimental procedures are displayed in Sect. 2. with the corresponding results. The constitutive models and the numerical procedures are presented in Sect. 3. The given results are then compared to experiments in Sect. 4.

\section{Materials and experiments}

\subsection{Materials and samples}

The $\mathrm{Zr}_{55} \mathrm{Cu}_{30} \mathrm{Al}_{10} \mathrm{Ni}_{5}$ (at. \%) BMG is studied in this paper. By a very careful tilt-casting process and with highly purified (50 ppm of oxygen) iodine refining processed $\mathrm{Zr}$, specimens with less than $300 \mathrm{ppm}$ of oxygen and no micrometric crystalline defects are prepared (see [15] for details). The material is supplied in the form of $60 \mathrm{~mm}$ long and $8 \mathrm{~mm}$ diameter rods. The samples used are cut and polished to obtain 6 or $15 \mathrm{~mm}$ long cylinders.

Experiments were carried out at room temperature and atmospheric pressure employing a hydraulic tension-compression machine (INSTRON 8803) equipped with a $500 \mathrm{kN}$ capacity load cell with a $8 \mathrm{~N}$ resolution. A $0.8 \mu \mathrm{m}$ resolution mechanical extensometer was used to measure the global displacement between the compression anvils, and two cameras were 
installed to monitor the displacement (with a $0.25 \mathrm{~mm}$ resolution) of a cloud of dots sprayed on a side of the sample (see Figure 1), and further derived the related strain field using a Digital Image Correlation (DIC) software [16] (Aramis). The tests were carried out at a displacement rate of $0.05 \mathrm{~mm} \cdot \mathrm{min}^{-1}$. For questions of reproducibility, the same test was made on four different samples times from the same batch. The load-displacement curves were the same within less than $5 \%$.

An equivalent shear strain used in this paper is defined as :

$$
\varepsilon^{e q}=\sqrt{\frac{2}{3} \underset{\sim}{e}: \underset{\sim}{e}}
$$

Where $\underset{\sim}{e}$ is the deviatoric part of the strain tensor $\underset{\sim}{\varepsilon}$ and ":" denotes the doubley contracted product.

\subsection{Experimental results}

The load-displacement (P-u) curve is presented in Fig. 2. The carried out test is a sequence of two successive loadings: the first one on a $15 \mathrm{~mm}$ sample, and the second one on the same sample cut to $6 \mathrm{~mm}$ long, after unloading of the former, to be able to reach fracture without damaging the compression anvils (100 kN limit load). Therefore P stands for a load per unit length. On Fig. 2 one can clearly distinguish two distinctive steps during the deformation process, a stiff part followed by a more compliant one. The numerical results presented in section 4 provide an explanation to this phenomenon. A higher magnification of the last part of the plot displays the presence of regularly spaced serrations, already reported elsewhere for different tests $[13,17]$. They are assumed to be the consequences of the sequential formation and emergence of shear bands inside and at the surface of the sample.

DIC allows one to capture the deformation pattern of the sample (see Fig. 3). During the first part of the loading (below $\sim 11 \mathrm{kN} / \mathrm{mm}$ ) it concentrates in a column underneath the load with a maximum equivalent strain of $\sim 0.2$ close to the anvil (indentation-like feature) and at the centre of the sample (Brazilian-like feature). At the end of the second loading, before fracture, most of the deformation fields are localised in a cross with a maximum intensity reached in its centre. The equivalent strain in this cross is more than 0.3 , while in the centre the deformation is higher than 0.6. These strains are average values since the shear bands thickness is 10 to $100 \mathrm{~nm}$ [18] while the DIC's resolution is $0.25 \mathrm{~mm}$. Much larger strains are expected inside the shear bands.

\section{Constitutive modelling and numerical simulations}

\subsection{Principles of the different approaches}

Metallic glasses are often compared to polycrystalline metallic alloys for their chemical composition and atomic bonds, with polymers for their behaviour at high temperature [19] and with ceramics for their brittleness [20]. Some models exist aiming at describing each of these materials behaviour, yet for amorphous metals their number is much smaller because there remain many unknowns in the understanding of the mechanisms governing their deformation. One can quote two specific theories proposed in the late seventies and which are references in metallic glasses behaviour: Spaepen's theory of free volume [5] and Argon's theory of shear transformation zones [4]. Depending on the underlying mechanisms 
assumed to be at the origin of the BMG deformation and on the final application, different state variables have to be accounted for in the constitutive modelling. For this reason, our modelling approach consisted in starting from very simple models and progressively increase their complexity. The constitutive models used in the following are accordingly: von Mises (VM) plasticity model involving the deviatoric stress; Drucker-Prager (DP) model involving the pressure in addition to the latter (some indentation studies found this model to be quite suitable $[21,10,11]$ ); the FV theory described by Gao [6] and AS model [7]. There exist several other models that have not been considered here [22, 23, 24].

\subsection{Constitutive equations}

The main equations describing each model are presented here. Let consider beforehand the decomposition of the total strain rate $\underset{\sim}{\dot{\varepsilon}}$ into a reversible part, namely $\dot{\mathcal{\varepsilon}}^{e}$, and an inelastic part, namely $\dot{\varepsilon}^{p}$. In the small strains assumption :

$$
\dot{\sim}=\dot{\sim}^{e}+\dot{\sim}^{p}
$$

In the context of linear isotropic elasticity, the following relationship holds:

$$
\dot{\sim}^{e}=\frac{1+v}{E} \dot{\sim}-\frac{v}{E}(\operatorname{tr} \underset{\sim}{\dot{\sigma}}) \underset{\sim}{\delta}
$$

where $\sigma$ represents the second order stress tensor. Note that $\sigma=\underset{\sim}{s}-p \underset{\sim}{\delta}$, with $\underset{\sim}{s}$ being the deviatoric part of $\sigma, p$ the pressure and $\delta$ the second order identity tensor. $E$ and $v$ are the Young's modulus and Poisson's ratio, respectively. The absence of strain hardening [1] lead us to use DP and VM as elastic perfectly plastic models (associated flow). This effect may be described using von Mises yield criterion which is written as:

$$
f_{M}\left(\sigma_{e q}\right)=\sigma_{e q}-\sigma_{0} \leq 0
$$

where $\sigma_{e q}=\sqrt{\frac{3}{2} \underset{\sim}{s}: \sim_{\sim}^{s}}$ represent the equivalent shear stress, $\sigma_{0}$ being the tensile yield strength. The asymmetry observed on BMG behaviour between yield strengths in tension and compression before failure may be described using the pressure dependent DP model widely used in soils mechanics:

$$
f_{D P}\left(\sigma_{e q}, p\right)=\sigma_{e q}-p \tan \varphi-\sigma_{0} \leq 0
$$

where $\varphi$ is the internal friction angle.

The next two models are specifically dedicated to amorphous materials with the purpose of representing inhomogeneous deformation under the form of localised shear bands. These works are inspired from two theories born in the late seventies. They argued that the specific behaviour of amorphous metals founds its origins at the atomic scale. Spaepen [5] assumed that the deformation is intimately related to the evolution of the free volume appearing during the cooling process leading to the formation of the amorphous metal. The free volume is frequently defined as the difference in volume between a crystalline alloy and its amorphous equivalent. The theory proposed by Argon [4] states that the deformation occurs by local shearing of clusters of atoms. According to his theory, tens of atoms slip on each other leading to local intense shearing. This phenomenon was reproduced using computational simulations aiming at describing interactions at the atomistic scale [25]. The two models 
presented hereafter have no yield criteria, the plastic strain is always present, but negligible during the so-called elastic part of the deformation.

For the FV model [6], the inelastic strain rate is written as:

$$
\dot{\sim}^{p}=\exp \left(-\frac{1}{v_{f}}\right) \sinh \left(\frac{\sigma_{e q}}{\sigma_{r e f}}\right) \frac{\stackrel{s}{\sim}}{\sigma_{e q}}
$$

where $v_{f}$ represents the free volume whose evolution law is given by:

$$
\dot{v}_{f}=\frac{1}{\alpha} \exp \left(-\frac{1}{v_{f}}\right)\left\{\frac{3(1-v)}{E}\left(\frac{\sigma_{r e f}}{\chi v_{f}}\right)\left[\cosh \left(\frac{\sigma_{e q}}{\sigma_{r e f}}\right)-1\right]-\frac{1}{n_{D}}\right\}
$$

where $\sigma_{r e f}$ is the reference stress, $\alpha$ is a geometrical factor, $\chi$ is a constant and $n_{D}$ is the number of atomic jumps needed to annihilate a free volume.

The last model considered in the present work is proposed by Anand and Su [7]. It aims at reproducing the elastic-viscoplastic response of pressure-sensitive and plastically-dilatant isotropic materials. The slip systems $\left(\underline{m}^{(\alpha)}\right.$ and $\underline{g}^{(\alpha)}$ are the normal to the $\alpha^{\text {th }}$ slip plane and its slip direction, respectively) are assumed to be related to the principal directions of stress, giving six of them in an isotropic material. The slip directions also depend on the angle of internal friction. The inelastic strain rate takes the form:

$$
\begin{gathered}
\stackrel{\dot{\epsilon}}{ }^{p}=\sum_{\alpha=1}^{6} \dot{\gamma}^{(\alpha)}\left\{G_{q^{(\alpha)}}+\beta M_{\sim}^{(\alpha)}\right\} \\
\underline{\sim}^{(\alpha)}=\operatorname{sym}\left[\underline{g}^{(\alpha)} \otimes \underline{m}^{(\alpha)}\right] \\
\underline{\sim}^{(\alpha)}=\underline{m}^{(\alpha)} \otimes \underline{m}^{(\alpha)}
\end{gathered}
$$

Where $\alpha$ represents one of the slip systems considered ( $\otimes$ is the dyadic tensor product). $\beta$ is the dilatancy parameter that controls the plastic flow direction:

$$
\beta=g_{0}\left(1-\frac{\eta}{\eta_{c v}}\right)^{p}
$$

The initial value of $\beta$ is $g_{0}$ thus the inelastic strain is a combination of plastic slip and dilatation; and tends to zero when the plastic volumetric strain $\eta$ (Eq. 10) reaches its limit $\eta_{c v}$ (the maximum density reduction as a result of plastic deformation) leading to plastic slip without dilatation. $g_{0}$ is a parameter that allows to dissociate the tension $\left(g_{0 t}\right)$ and compression $\left(g_{0 c}\right)$ behaviour. The plastic volumetric strain rate $(\dot{\eta})$ reads $(\dot{\gamma}$ is the total shearing rate):

$$
\dot{\eta}=\beta \dot{\gamma}
$$

Let $\dot{\gamma}^{(\alpha)}$ be the shearing rate on the slip system $\alpha$. It is expressed via a viscoplastic Norton-like law with a Mohr-Coulomb type resistance coefficient:

$$
\begin{gathered}
\dot{\gamma}^{(\alpha)}=\dot{\gamma}_{0}\left(\frac{\tau^{(\alpha)}}{c+\mu \sigma^{(\alpha)}}\right)^{1 / m}>0 \\
\dot{\gamma}=\sum_{\substack{\alpha=1 \\
5}}^{6} \dot{\gamma}^{(\alpha)}
\end{gathered}
$$


Where $\tau^{(\alpha)}=\sigma: G_{\sim}^{(\alpha)}$ and $\sigma^{(\alpha)}=-\sigma: M_{\sim}^{(\alpha)}$ represent the resolved shear stress and the normal stress on the slip system $\alpha$, respectively. The quantity $\dot{\gamma}_{0}$ is a reference shearing rate. $m$ is the strain-rate sensitivity, taken small for a nearly rate independent response. The constant $\mu$ is the internal friction coefficient. The evolution law of the cohesion $c$ is taken in the form:

$$
\begin{gathered}
c=c_{c v}+b\left(1-\frac{\eta}{\eta_{c v}}\right)^{q} \\
c(0)=c_{c v}+b
\end{gathered}
$$

Where $c(0)$ is the initial cohesion, composed of the final cohesion $c_{c v}$ with an overshoot $b$. The parameters $q$ and $p$ are positive material constants of order unity.

\subsection{Numerical procedures}

The engineering Finite Element computation code Abaqus was used to conduct the computational simulations. VM and DP models belong to the material library of the code. FV and AS models (see Sec. 3) were implemented as user-material in Abaqus. The calculation is first made in the corotational framework via equations presented in Sec. 3.2, then the software takes into account the finite strains. Taking advantage of the symmetries of the boundary value problem, the sample is modelled by a quarter of circle composed with 4-nodes finite elements with reduced integration and assuming plane strain conditions (CPE4R). The compression anvil is modelled using an analytical rigid part and is submitted to a displacement at constant velocity with a frictionless contact.

The different coefficients used for the simulations are given in Tab. 1-4. The elastic constants have been determined using ultra-sound techniques [26]. For DP model, the yield strengths were chosen corresponding to the material's behaviour [27]. For VM model, the mean value of the two was used. Those two are elastic perfectly plastic models since there is no strain hardening in the material. For FV and AS models, the coefficients are taken from literature $[6,7]$ and correspond to a Zr-based BMG.

\subsection{Identification of material parameters}

With the aim of obtaining a better match between experimental and numerical results, an automated material parameters identification procedure based on the hybrid method (Levenberg-Marquardt, gradient and Newton-Raphson algorithms) [28, 29] is used. In doing so, only a fraction of the parameters (see Tab. 1-4) has been allowed to vary. Namely for DP model: the compressive and tensile yield stresses, $\sigma_{0 c}$ and $\sigma_{0 t}$ respectively; for AS model: the cohesion and the internal friction, $c$ and $\mu$ respectively.

\section{Computational results and discussion}

The computational simulation offers the possibility to observe the inner stress fields, unavailable experimentally. The origin of the inflection point (Fig. 2 at $\sim 7 \mathrm{kN} \cdot \mathrm{mm}^{-1}$ ) can then be linked to the coalescence of the high stress fields up and down the sample (Fig. 4). The fields of plastic strain developed at the contact join, forming a constant vertical field. Besides, one can notice the von Mises stress decreasing from the centre to the sides which are almost free of loading. That is why the shear bands do not propagate in this area (as we can observe numerically on Fig. 5). 
The equivalent strain map obtained with FV (see Fig. 5) or AS models localise the strain in fine numerical shear bands [30]. Note that the simulations were conducted with a very fine meshing (120.000 elements) using a variable free volume for FV model and a variable cohesion for AS model. The shear bands directions obtained numerically correspond to those observed experimentally, their number and intensity obviously depend on the mesh size. Corresponding to each serration, one can notice (not shown here) on the deformation pattern the creation of a new band with high deformation. The magnitude of the serrations is closely related to the mesh size. The finer the latter, the smoother the former. This the drawback of models with softening like AS and FV: local fields depend on the mesh size and therefore can not be compared one-to-one to experiments. Let us point out finally that the computational simulations were made in plane strain conditions while the experimental data gave a plane stress condition map. Nevertheless, it appears that the most intense numerical shear band is one branch of the pattern observed experimentally (Fig. 3). The resolution obtained with the DIC device does not allow us to observe thin shear bands like in the numerical results, but a semi-local map of the deformation process.

The superposition of the experimental and numerical overall load-displacement curves are plotted in Fig. 6. From a qualitative point of view, the curves obtained with the VM and DP models follow an evolution similar to the experiment. Before the inflection point VM and DP models have a slope very close to the experimental one, while the amorphous dedicated models overestimate it. After this point, the slopes of the remaining curve are the same at the beginning, except for FV model. The latter exhibits a plateau which does not exist experimentally. For higher deformations, the experimental load grows faster than the numerical ones.

Optimizing the plasticity coefficients for DP and AS models (they have proven to be much better than the other models studied) has given interesting results (Fig. 7). The first part of the curve (label 1) is well described by DP, and the slope is a little overestimated by AS. For the second part (label 2), the slopes obtained are the same, the reloading is well described by the two models. On the third part (label 3) one can notice that the slopes of the two models slightly underestimate the experiments. The results obtained with those models give a possible set of plasticity coefficients presented in Tab. 5. The simulations not taking into account the stiffness of the compression anvils, the coefficients obtained are probably underestimated, and so represent a first approach before the use of a more accurate numerical procedure. As for DP model the parameters differ from those obtained by indentation techniques [21].

In terms of local information, and this is a key point of this paper, it is highlighted in Fig. 5 that two models that predict load-displacement curves very similar (see Fig. 2 give very different deformation fields.

\section{Concluding remarks}

In this study, we have carried out diametral compression or Brazilian tests on a Zr-based BMG. This test allows us to get an insight on the plastic behaviour of the material, unlike conventional tests (tension, compression) because of the brittleness of the material. The present approach differs from previous constrained tests studies such as indentation since it allows to record in situ extensive plastic fields during the test, via Digital Image Correlation techniques (local information), in addition to the mean response (the load-displacement curve, global information). Besides, for the indentation test, the stress fields are highly 
compressive and localised underneath the indenter tip, while for the Brazilian test, they are compressive close to the loading conditions, tensile close to the free boundaries and close to pure at the centre. Intense plasticity was found to develop in the sample, first like in an indentation test, then localised in a cross-like pattern. Values of the equivalent strain, prior to failure, was as high as more than 0.8 , in the centre of this cross.

Through the use of constitutive models with growing complexity, we have observed that all models may reproduce the global information (load-displacement curve) with identified material parameters. However, they predict different contrasted levels of the local information (equivalent strain).

The search for a relevant model able to describe the plasticity of metallic glasses under various mechanical loadings will therefore require to take into account this local information, as well as other constrained tests like indentation or scratching [31] and uniaxial tests (yield strengths).

\section{Acknowledgements}

Jean-Sébastien Brest acknowledges the Brittany region (ARED CODYVER) and DGA for his PhD grant ; the European Brittany University (EPT COMDYNVER, avec le soutien du Fonds Européen de Développement Régional) and the Brittany region (DCICOLL) for their financial support as well as Pr P. Pilvin for fruitful discussion.

\section{References}

[1] C. A. Schuh, T. C. Hufnagel, U. Ramamurty, Acta Mater. 55 (12) (2007) 4067-4109.

[2] J. Xu, U. Ramamurty, E. Ma, JOM 62 (4) (2010) 10-18.

[3] V. Keryvin, Y. Nadot, Y. Yokoyama, Scripta Mater. 57 (2) (2007) 145-148.

[4] A. S. Argon, Acta Metall. 27 (1) (1979) 47-58.

[5] F. Spaepen, Acta Metall. 25 (4) (1977) 407-415.

[6] Y. F. Gao, Modell. Simul. Mater. Sci. Eng 14 (8) (2006) 1329-1345.

[7] L. Anand, C. Su, J. Mech. Phys. Solids 53 (6) (2005) 1362-1396.

[8] V. Keryvin, Acta Mater. 55 (8) (2007) 2565-2578.

[9] C. A. Schuh, A. C. Lund, T. Nieh, Acta Mater. 52 (20) (2004) 5879-5891.

[10] M. Patnaik, R. Narasimhan, U. Ramamurty, Acta Mater. 52 (11) (2004) 3335-3345.

[11] U. Ramamurty, S. Jana, Y. Kawamura, K. Chattopadhyay, Acta Mater. 53 (3) (2005) 705-717.

[12] R. D. Conner, W. L. Johnson, N. E. Paton, W. D. Nix, J. Appl. Phys. 94 (2) (2003) 904.

[13] W. H. Jiang, F. X. Liu, P. K. Liaw, H. Choo, Appl. Phys. Lett. 90 (18) (2007) 181903.

[14] M. Mellor, I. Hawkes, Eng. Geol. 5 (3) (1971) 173-225.

[15] Y. Yokoyama, K. Fukaura, A. Inoue, Intermetallics 10 (11-12) (2002) 1113-1124.

[16] T. Chu, W. Ranson, M. Sutton, Exp. Mech. 25 (3) (1985) 232-244.

[17] S. X. Song, T. G. Nieh, Intermetallics 17 (9) (2009) 762-767.

[18] T. C. Hufnagel, P. El-Deiry, R. P. Vinci, Scripta Mater. 43 (12) (2000) 1071-1075.

[19] K. E. Prasad, V. Keryvin, U. Ramamurty, J. Mater. Res. 24 (03) (2009) 865-872.

[20] V. Keryvin, V. H. Hoang, J. Shen, Intermetallics 17 (4) (2009) 211-217.

[21] V. Keryvin, J. Phys.: Cond. Matter 20 (11) (2008) 114119.

[22] P. Thamburaja, R. Ekambaram, J. Mech. Phys. Solids 55 (6) (2007) 1236-1273.

[23] M. Zhao, M. Li, J. Mater. Res. 24 (8) (2009) 2688-2696.

[24] Q. Yang, A. Mota, M. Ortiz, Comput. Mech. 37 (2) (2006) 194-204.

[25] D. Deng, A. S. Argon, S. Yip, Philos. Trans. Roy. Soc. A 329 (1608) (1989) 613-640.

[26] V. Keryvin, T. Rouxel, M. Huger, L. Charleux, J. Ceram. Soc. Jpn. 116 (1356) (2008) 851-854.

[27] V. Keryvin, K. E. Prasad, Y. Gueguen, J. C. Sangleboeuf, U. Ramamurty, Phil. Mag. 88 (12) (2008) 1773-1790.

[28] G. Cailletaud, P. Pilvin, ASME 43 (1993) 33-45.

[29] A. Andrade-Campos, S. Thuillier, P. Pilvin, F. Teixeira-Dias, Int. J. Plasticity 23 (8) (2007) 1349-1379. 
[30] C. Su, L. Anand, Acta Mater. 54 (1) (2006) 179-189.

[31] V. Keryvin, R. Crosnier, R. Laniel, V. H. Hoang, J.-C. Sanglebœuf, J. Phys. D: Appl. Phys. 41 (7) (2008) 074029. 


\section{List of Figures}

1 Schematics of the experimental set-up and its instrumentation. . . . . . . . 11

2 Diametral compression results - Plot of load per unit length vs displacement Magnification of the serrations at the end of the curve. . . . . . . . . . . 11

3 Map of equivalent strain (logarithmic measure $\log \left(1+\varepsilon^{e q}\right)$ ) obtained by DIC ; at the end of the first loading ( $\sim 11 \mathrm{kN} \cdot \mathrm{mm}^{-1}$, top) and at the end of the second loading up to fracture (bottom, strain reinitialised to zero). . . . . . . 12

4 Mises stress fields obtained numerically below the inflection point of the curve in Fig. 2 with VM model. . . . . . . . . . . . . . . . . . . . . . . . 13

5 Map of equivalent strain obtained for a $2 \mathrm{~mm}$ displacement: DP model (left) and FV model (right). In this latter case, the whole sample was meshed. The material parameters used are presented in Tab. 1-4. Note that a similar colormap was used for comparing. . . . . . . . . . . . . . 14

6 Comparison between experimental and numerical results using the material parameters presented in Tab. 1-4. . . . . . . . . . . . . . . . . . . . 15

7 Comparison of the experimental and numerical results, after reverse identification on DP and AS models. . . . . . . . . . . . . . . . . . . . . 16 


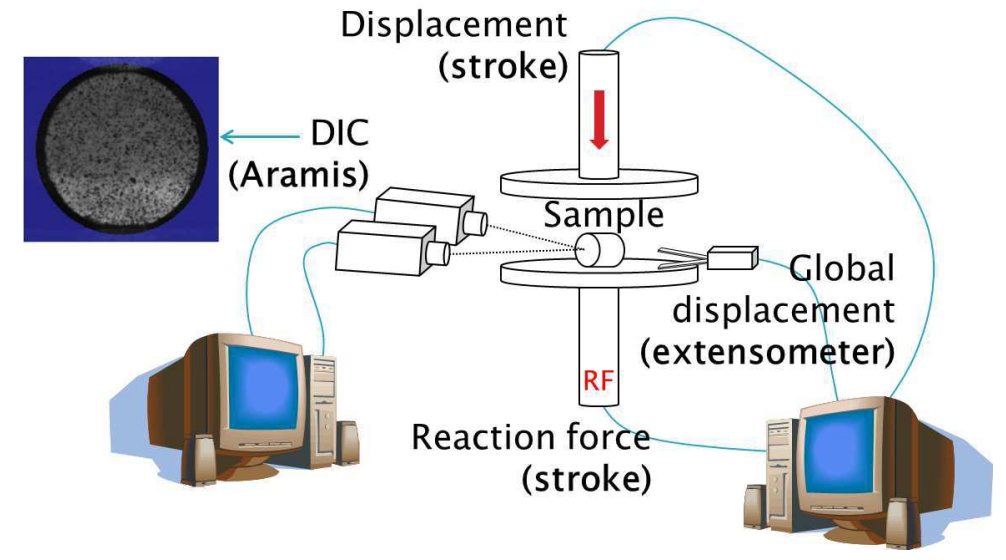

Figure 1: Schematics of the experimental set-up and its instrumentation.

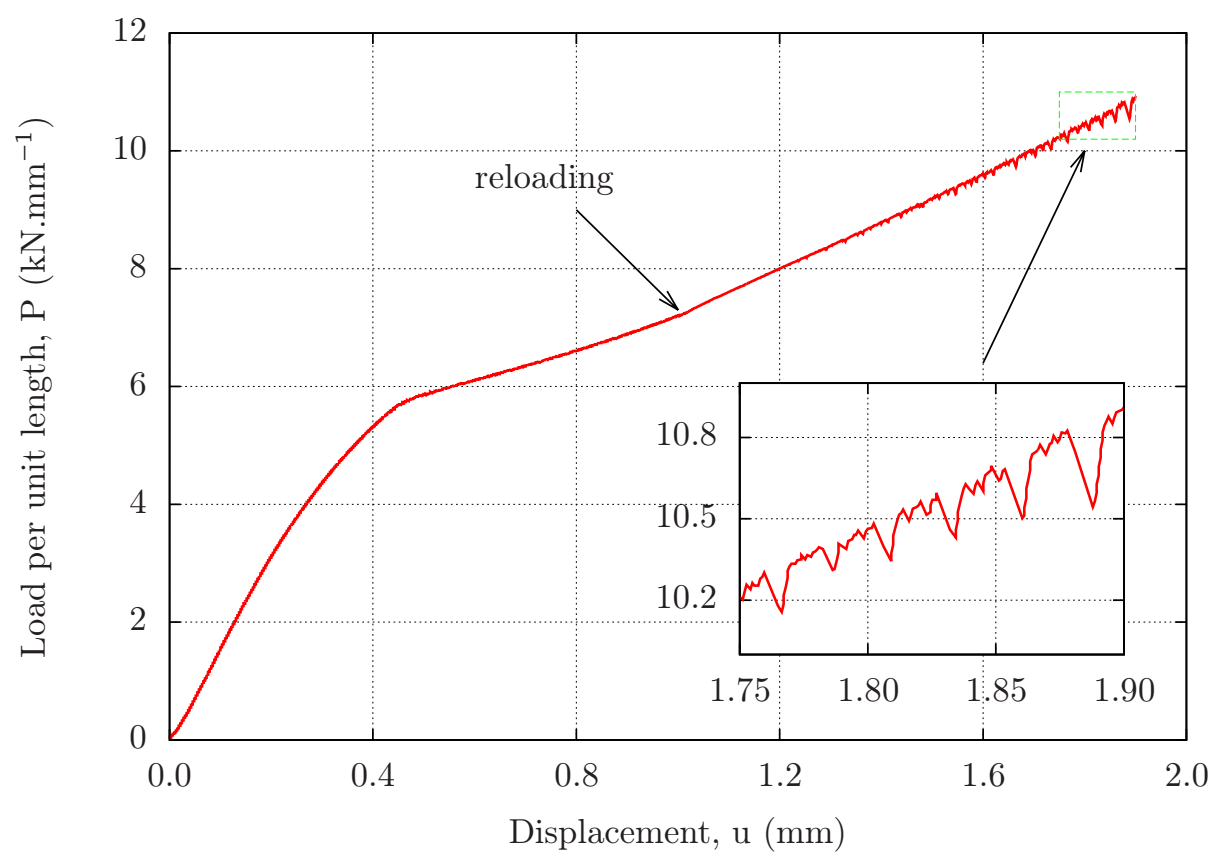

Figure 2: Diametral compression results - Plot of load per unit length vs displacement - Magnification of the serrations at the end of the curve. 

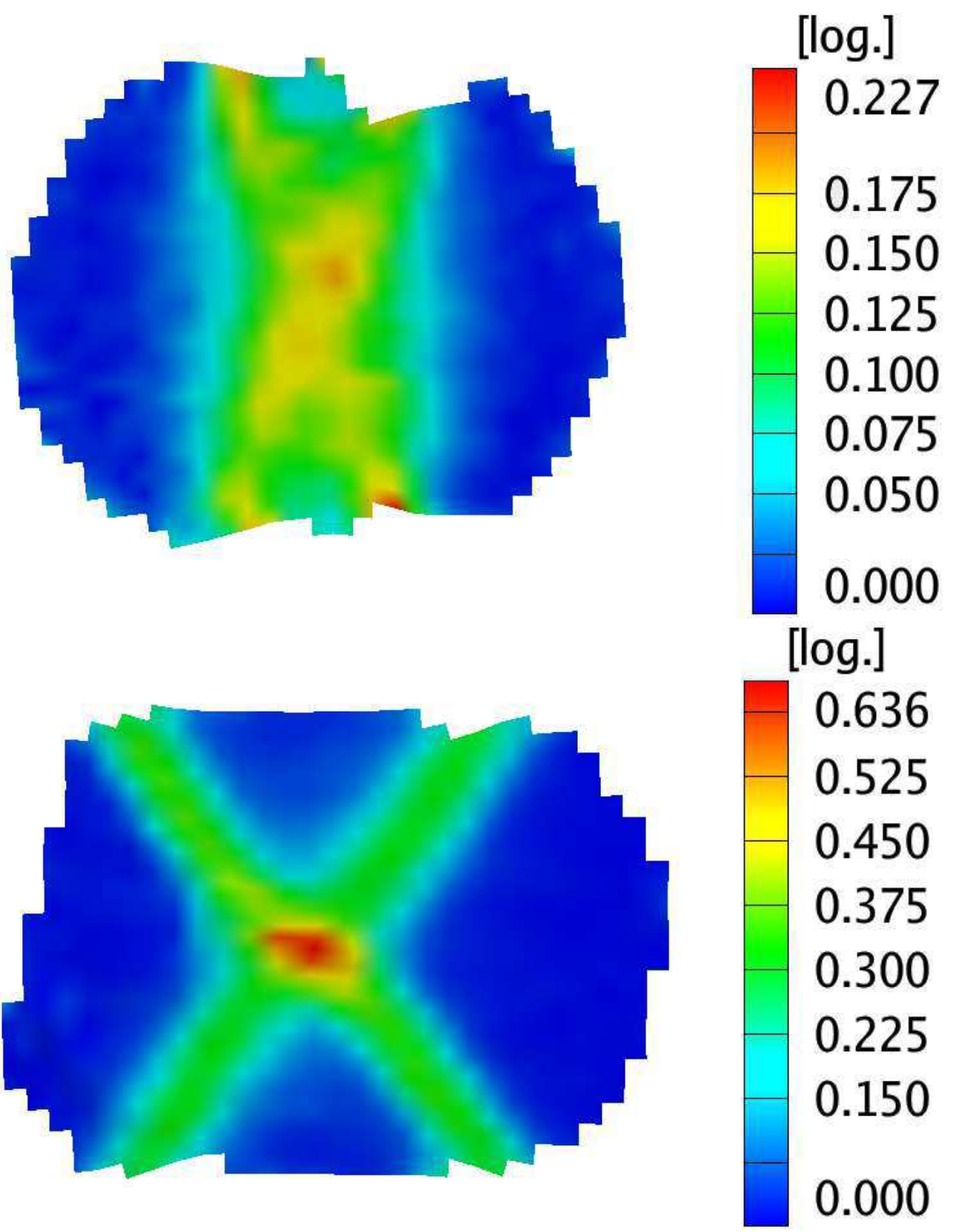

Map of equivalent strain (logarithmic measure) obtained by Digital Image Correlation on the $\mathrm{Zr}_{55} \mathrm{Cu}_{30} \mathrm{Al}_{10} \mathrm{Ni}_{5}$ under diametral compression (Brazilian disk) ; at the end of the first loading ( $\sim 11 \mathrm{kN} \cdot \mathrm{mm}^{-1}$, top) and at the end of the second loading up to fracture (bottom, strain reinitialised to zero)

Figure 3: Map of equivalent strain (logarithmic measure $\log \left(1+\varepsilon^{e q}\right)$ ) obtained by DIC ; at the end of the first loading ( $\sim 11 \mathrm{kN} \cdot \mathrm{mm}^{-1}$, top) and at the end of the second loading up to fracture (bottom, strain reinitialised to zero). 


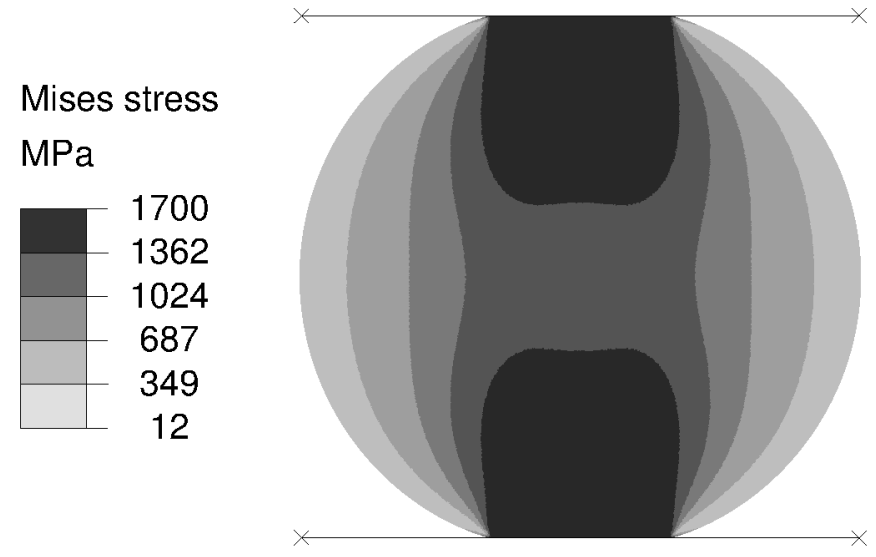

Figure 4: Mises stress fields obtained numerically below the inflection point of the curve in Fig. 2 with VM model. 

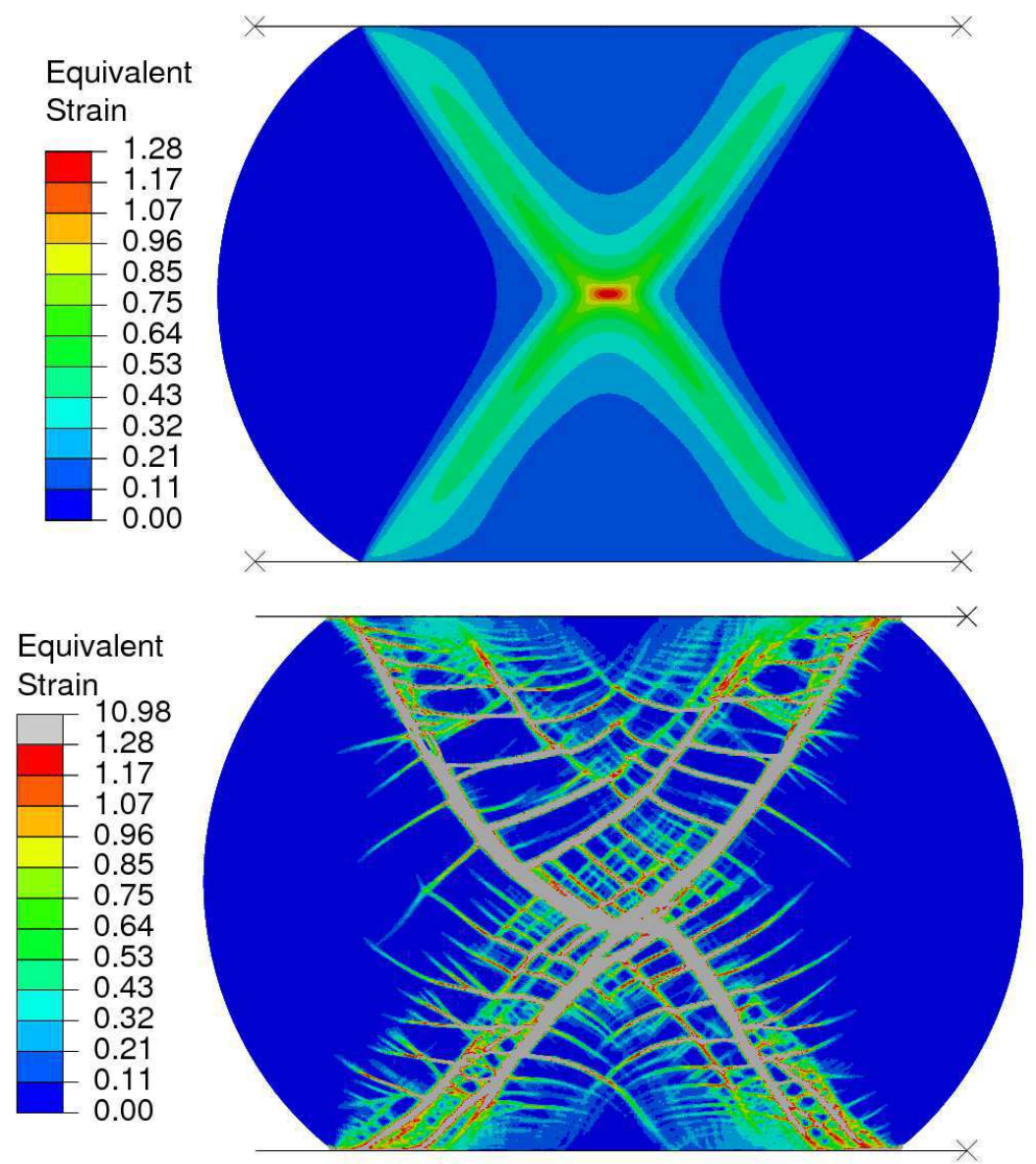

Figure 5: Map of equivalent strain obtained for a $2 \mathrm{~mm}$ displacement: DP model (left) and FV model (right). In this latter case, the whole sample was meshed. The material parameters used are presented in Tab. 1-4. Note that a similar colormap was used for comparing. 


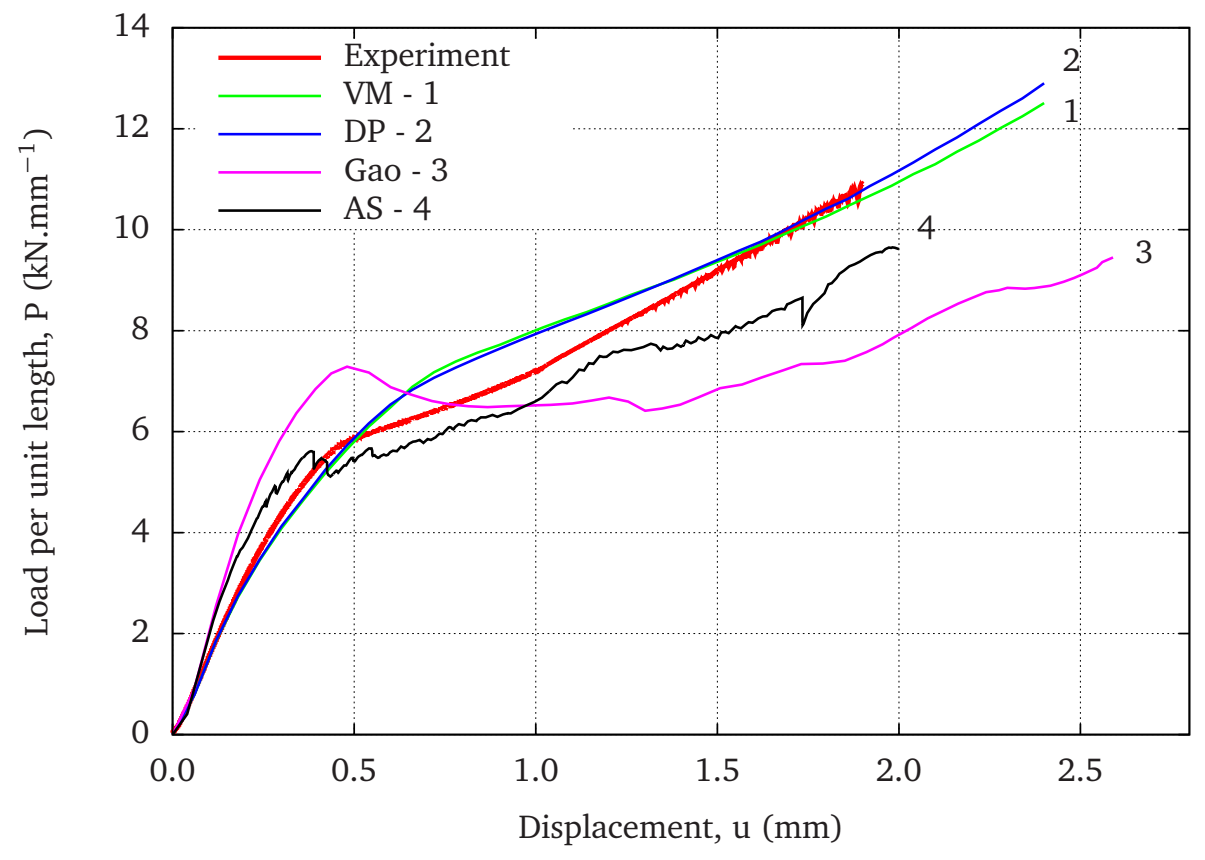

Figure 6: Comparison between experimental and numerical results using the material parameters presented in Tab. 1-4. 


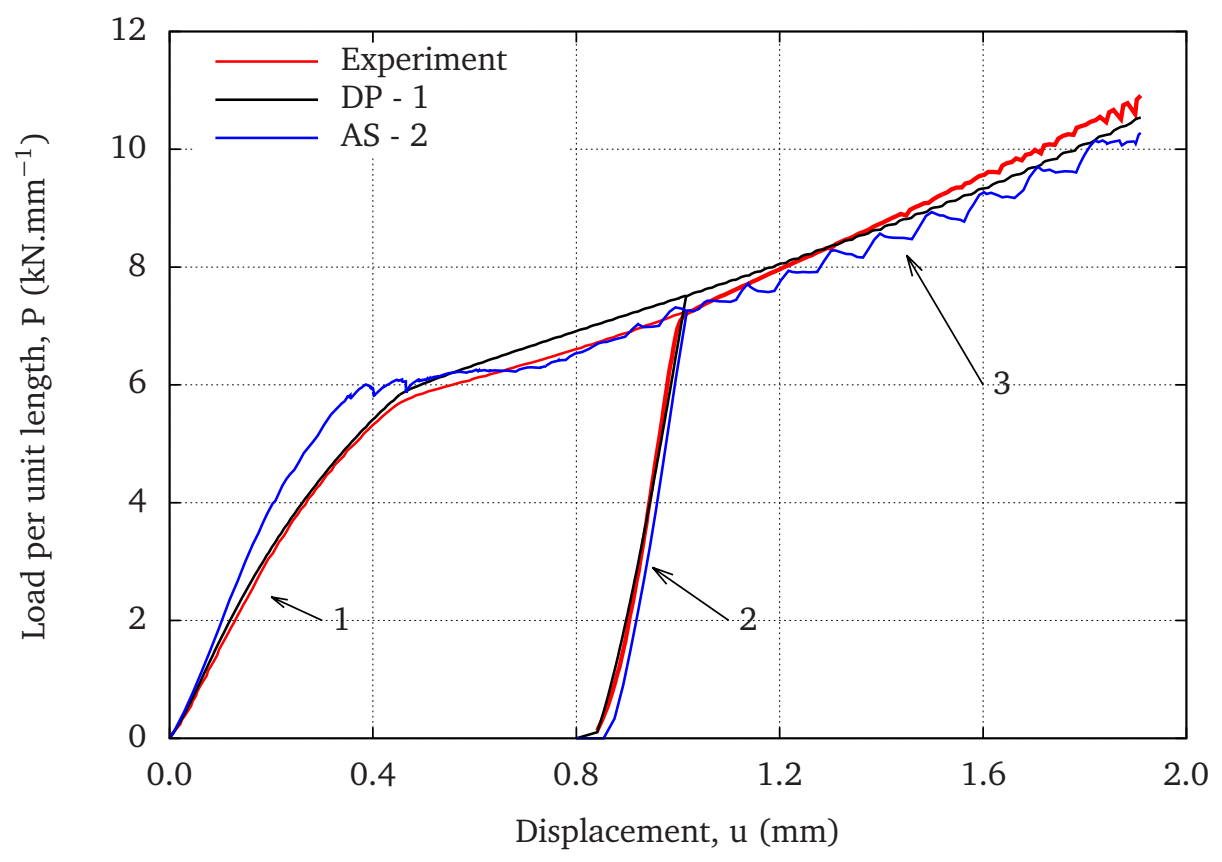

Figure 7: Comparison of the experimental and numerical results, after reverse identification on DP and AS models. 


\section{List of Tables}

1 Materials parameters used for VM model. . . . . . . . . . . . . . . . 18

2 Materials parameters used for DP model. . . . . . . . . . . . . . . . . . . 19

3 Materials parameters for FV model. . . . . . . . . . . . . . . . . . . . . . . . . . . . .

4 Materials parameters used for AS model. . . . . . . . . . . . . . . . . . 21

5 Materials parameters obtained after identification. . . . . . . . . . . . . . 22 


$$
\begin{array}{c|c|c}
E(\mathrm{MPa}) & v(-) & \sigma_{0}(\mathrm{MPa}) \\
\hline 85000 & 0.37 & 1700
\end{array}
$$

Table 1: Materials parameters used for VM model. 


\begin{tabular}{c|c|c|c}
$E(\mathrm{MPa})$ & $v(-)$ & $\sigma_{0 t}(\mathrm{MPa})$ & $\sigma_{0 c}(\mathrm{MPa})$ \\
\hline 85000 & 0.37 & 1600 & 1800
\end{tabular}

Table 2: Materials parameters used for DP model. 


\begin{tabular}{c|c|c|c|c|c}
$E(\mathrm{MPa})$ & $v(-)$ & $\sigma_{\text {ref }}(\mathrm{MPa})$ & $n_{D}(-)$ & $\alpha(-)$ & $\chi(-)$ \\
\hline 85000 & 0.37 & 100 & 3 & 0.15 & 1
\end{tabular}

Table 3: Materials parameters for FV model. 


\begin{tabular}{c|c|c|c|c|c}
$E(\mathrm{MPa})$ & $v(-)$ & $\mu(-)$ & $\dot{\gamma}_{0}\left(s^{-1}\right)$ & $m(-)$ & $g_{0 c}(-)$ \\
\hline 85000 & 0.37 & 0.04 & 0.001 & 0.005 & 0.04 \\
\hline \hline$g_{0 t}(-)$ & $\eta_{c v}(-)$ & $p(-)$ & $c_{0}(\mathrm{MPa})$ & $b(\mathrm{MPa})$ & $q(-)$ \\
\hline 0.4 & 0.005 & 0.8 & 960 & 300 & 1.2
\end{tabular}

Table 4: Materials parameters used for AS model. 


\begin{tabular}{c|c||c|c}
\multicolumn{2}{c||}{ Drucker Prager } & \multicolumn{2}{c}{ Anand } \\
\hline$\sigma_{y t}(\mathrm{MPa})$ & $\sigma_{y c}(\mathrm{MPa})$ & $c(\mathrm{MPa})$ & $\mu(-)$ \\
\hline 1300 & 1760 & 1080 & 0.057
\end{tabular}

Table 5: Materials parameters obtained after identification. 